Background: Chondrocalcinosis is a painful rheumatic condition caused by the deposition of calcium pyrophosphate dihydrate crystals (CPPD) in joint tissues, and especially in cartilage. It is known that CPPD crystals cause inflammation and degenerative changes in joint, but the underlying mechanisms remain poorly understood. In particular, nothing is known about how these crystals regulates transmembrane heparan sulphate proteoglycans (HSPGs). Our attention focused on one family of HSPGs called syndecans as they have important roles both as adhesion molecules, by mediating chondrocyte-extracellular matrix interactions, and as modulators of intracellular signaling triggered by cytokines and growth factors.

Objectives: The aim of this study was to evaluate how CPPD crystals modulates syndecan expression in chondrocytes and in cartilage, and how this modulation can be ultimately linked to cartilage damage during chondrocylcinosis.

Methods: Murine chondrocitic ATDC5 cells were stimulated with $0,1 \mathrm{ng} / \mathrm{ml}$ CPPD crystals or with $0,1 \mathrm{ng} / \mathrm{ml}$ basic-calcium phosphate crystals (BCP), a family of calcium-containing crystals found in other rheumatic conditions such as osteoarthritis (OA). Cytotoxicity was evaluated by lactate dehydrogenase (LDH) release in the supernatant at 30 minutes, and 3, 6, 24 hours after stimulation. At the same time-points, mRNA expression levels of syndecans (Synd-1, -2, -3, -4) and of matrix-degrading enzymes (Mmp-3, -9, -13; Adamts-4, -5) was analysed by qRT-PCR. Finally, Syndecan-4 protein expression was studied by immunohistochemistry $(\mathrm{IHC})$ in cartilage samples of patients with chondrocalcinosis and in samples of patients with severe OA without chondrocalcinosis as control.

Results: LDH assay revealed no increased cytotoxicity by CPPD or BCP at any time-point. qRT-PCR indicated that CPPD crystals but not BCP crystals induced Synd-2 and -3 upregulation at 30 minutes after stimulation and Synd-4 upregulation at 3 hours, while no modulation of syndecans was seen at later time-points. CPPD also induced Adamts- 4 expression at 3 hours after stimulation, and Mmp- 9 expression at 3 and 6 hours. The expression of the other matrix-degrading enzymes was not affected. Human chondrocalcinosis cartilage exhibited enhanced Synd-4 expression compared to severe OA cartilage containing BCP calcification. Interestingly, Synd-4 expression was observed in the extracellular matrix but not on cell membrane, suggesting that maybe Synd-4 undergoes shedding (Figure 1)
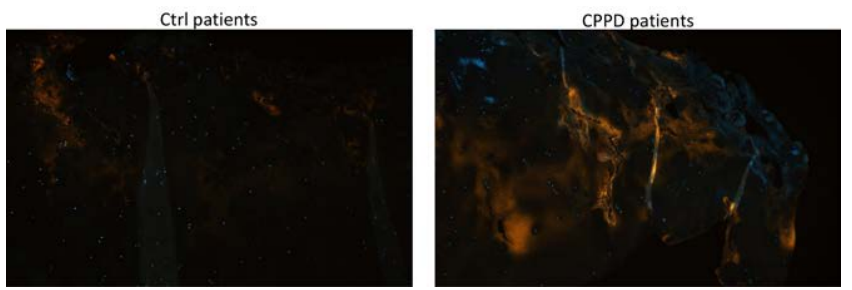

Figure 1. Representative Synd-4 IHC in control patients (Ctrl, severe OA) and chondrocalcinosis patients (CPPD). Note increased Synd-4 expression in extracellular matrix of CPPD patients compared to Ctrl ones.

Conclusion: BCP and CPPD crystals seem to trigger differential effects in terms of modulation of syndecans in chondrocitic cells. CPPD crystals induce Synd-4 and Adamts- 4 and Mmp- 9 which are not induced by BCP crystals. It remains to be clarified whether the two events are interlinked. In particular, further studies are required to investigate if Adamts-4 and Mmp-9 are involved in Synd-4 shedding or if vice versa Synd-4 regulates Adamts-4 and Mmp-9 activation and downstream cartilage breakdown in chondrocalcinosis.

Disclosure of Interests: : Sonia Nasi: None declared, Jessica Bertrand Grant/ research support from: Pfizer, Speakers bureau: Pfizer, Miriam Bollmann: None declared, Richard Stange: None declared, Thomas Pap: None declared DOI: 10.1136/annrheumdis-2020-eular.2772

\section{THU0436 ASSESSMENT OF CARDIOVASCULAR RISK LEVELS IN CPPD VERSUS RA AND GOUT, AND RISK- FLUCTUATION ANALYSIS BASED ON CALCULATOR TYPE: ATP III AND REYNOLDS RISK SCORE}

A. Novikova ${ }^{1}$, M. Eliseev ${ }^{1}$, O. Sheliabina ${ }^{1}$, H. Gerasimova ${ }^{1} .{ }^{1}$ VA Nasonova Research Institute of Rheumatology, Moscow, Russian Federation

Background: Cardiovascular risk in CPPD patients is not so well evaluated as in other rheumatic diseases, and optimal risk calculators for patients with calcium pyrophosphate crystal deposition disease have not yet been studied.

Objectives: To assess CVR and compare stratification results using ATP III and Reynolds Risk Score (RRS) calculators in CPPD, RA and gout patients versus the control subjects.

Methods: The case-control study included 168 patients aged 18 - 80 years old, with 42 participants in each subgroup - CPPD, gout, RA patients and healthy volunteers, matched by gender (10 males and 32 females) and age (mean age 54 years). CPPD diagnosis was based on McCarty 1961 y criteria, RA - following ACR/EULAR 2010 y criteria, and gout - ACR/EULAR 2015 criteria. CPPD and gout diagnosis was crystal- verified in all cases. Exclusion criteria were as follows: diabetes mellitus and eGFR $<60 \mathrm{ml} / \mathrm{min} / 1.73 \mathrm{~m}^{2}$. The following data was collected for all patients: anthropometric parameters, BP, lab tests, including serum glucose level, creatinine, total cholesterol (TC), HDLp, CRP; CVR was assessed using ATP III and RRS scales. Statistica 12.0 package was used for statistical data processing.

Results: Both groups were comparable in terms of anthropometric parameters rates of individual indicators and factors did not differ, except for family history of cardiovascular disease, systolic BP, HDLp, hsCRP (see Table).

Table 1. Risk factors and CVR stratification by ATP III and RRS in CPPD, RA, gout and control group.

\begin{tabular}{|c|c|c|c|c|}
\hline & CPPD $(n=42)$ & $\mathrm{RA}(\mathrm{n}=42)$ & Gout $(n=42)$ & $\begin{array}{l}\text { Control } \\
(n=42)\end{array}$ \\
\hline Smoking, $\mathrm{n}(\%)$ & $11(26,2)$ & $12(28,6)$ & $8(19,0)$ & $12(28,6)$ \\
\hline Systolic $\mathrm{BP}, \mathrm{mmHg}, \mathrm{M}_{ \pm} \mathrm{SD}$ & $124 \pm 14^{* / * *}$ & $138 \pm 17^{\# \#}$ & $144 \pm 26^{\# \# \#}$ & $127 \pm 16$ \\
\hline $\mathrm{TC}, \mathrm{mg} / \mathrm{dl}, \mathrm{M} \pm \mathrm{SD}$ & $261.9 \pm 64.2$ & $244.1 \pm 77.5$ & $249.3 \pm 62.7$ & $244.1 \pm 52.6$ \\
\hline HDLp, mg/dl, $M_{ \pm} \mathrm{SD}$ & $63.2 \pm 20.2^{\star / \star \star}$ & $49.2 \pm 16.5^{\# \#}$ & $52.0 \pm 9.7^{\# \# \#}$ & $58.1 \pm 16.4$ \\
\hline $\begin{array}{l}\text { hsCRP, mg/l, Me }\left[25-75^{\text {th }}\right. \\
\text { percentiles] }\end{array}$ & 3. $\underset{* / \star \star / \star \star \star \star}{[1,0 ; 12,4]}$ & $8,6[4.1 ; 20.6]^{\# \#}$ & $8.5[4.1 ; 2.9]^{\# \# \#}$ & $1,5[0.8 ; 2.6$ \\
\hline $\begin{array}{l}\mathrm{hsCRP} \geq 5 \mathrm{mg} / \mathrm{l}, \\
\mathrm{n}(\%)\end{array}$ & $18(43)^{\star / \star \star \star / \star \star \star}$ & $27(64)^{\# \#}$ & $29(69)^{\# \# \#}$ & $3(7)$ \\
\hline Family history of CVD, $n \%$ & $6(14)^{\star / \star \star \star}$ & $16(38)^{\#}$ & $4(10){ }^{\# \# \#}$ & $17(40)$ \\
\hline $\begin{array}{l}\text { High and very high CVR } \\
\text { levels, ATP III scale } \\
(>10 \%), n(\%)\end{array}$ & $5(12)$ & $9(21)$ & $7(17)$ & $8(19)$ \\
\hline $\begin{array}{l}\text { High and very high CVR } \\
\text { levels, RRS scale (>10\%), } \\
\mathrm{n}(\%)\end{array}$ & $9(21)$ & $14(33)$ & $12(29)$ & $7(17)$ \\
\hline
\end{tabular}

$\# \#$ $<0.05$ between gout and controls.

Based on ATP III risk calculation the number of CPPD patients with high and very high CVR was $5(12 \%)$ patients and was close to that in RA $(9(21 \%))$, gout $(7$ $(17 \%))$ and the control group (8 (19\%)). Mean CRP levels and number of pts with $\mathrm{CRP} \geq 5 \mathrm{mg} / \mathrm{l}$ were significantly lower in CPPD and control group pts, than in RA and gout, however CRP $\geq 5 \mathrm{mg} / \mathrm{l}$ levels were documented almost in half of CPPD pts $(43 \%)$ and only in $7 \%$ of pts from the control group $(p<0.05)$.

Although CVR calculations based on RRS scale yielded similar results, and all groups remained comparable, nevertheless, the number of pts with high and very high CVR increased in each group, except for the control. There were no meaningful differences in between the groups in TC levels, however HDLp was significantly higher in CPPD pts $(p<0.05)$, than in RA and gout, and in the control group pts vs RA pts $(p<0.05)$

Conclusion: CPPD associated cardiovascular risk is considerably high and comparable to CVR levels in RA and gout. Given that RRS based CVR calculation resulted in increased number of patients with high and very high risks in all groups, except for the control group, it can be suggested that use of calculators including CRP is appropriate not only in RA pts, but also in microcrystal deposition arthritis, associated with inflammation, therefore prospective studies on larger samples are deemed necessary.

Disclosure of Interests: : Aleksandra Novikova: None declared, Maxim Eliseev Speakers bureau: Novartis, Menarini Group, Alium, Olga Sheliabina: None declared, Helen Gerasimova: None declared DOI: 10.1136/annrheumdis-2020-eular.5190

\section{THU0437 \\ SPECIFIC COMORBIDITIES ENHANCE MONOSODIUM URATE CRYSTAL DEPOSITION IN GOUT: A MULTICENTRE DUAL-ENERGY COMPUTED TOMOGRAPHY STUDY}

T. Pascart ${ }^{1}$, A. Ramon ${ }^{2}$, S. Ottaviani ${ }^{3}$, J. Legrand ${ }^{1}$, V. Ducoulombier ${ }^{1}$ E. Houvenagel ${ }^{1}$, L. Norberciak ${ }^{1}$, P. Richette ${ }^{4}$, F. Becce ${ }^{5}$, P. Ornetti ${ }^{2}$, J. F. Budzik ${ }^{1}$ ${ }^{1}$ Groupe Hospitalier de L'institut Catholique de Lille, Lille, France; ${ }^{2} \mathrm{CHU}$ Dijon, Dijon, France; ${ }^{3} \mathrm{Hoppital}$ Bichat, Paris, France; ${ }^{4} \mathrm{Hôpital}$ Lariboisière, Paris, France; ${ }^{5}$ CHUV Lausanne, Lausanne, Switzerland

Background: The reasons explaining why some patients exhibit higher monosodium urate (MSU) crystal burdens than others remain largely unknown. While MSU crystal formation is enhanced by certain factors in vitro such as $\mathrm{pH}$, temperature, and other ion concentrations, it is currently unknown whether comorbidities and clinical features are associated with increased MSU deposition in vivo. Objectives: To determine which factors are associated with the burden of MSU crystal deposition quantified by dual-energy CT (DECT) in urate lowering therapy (ULT)-naive gout patients.

Methods: In this multicenter cross-sectional study, DECT scans of knees and feet were prospectively obtained from ULT-naive, or not taking any ULT for more than a year, gout patients. Demographic, clinical (including gout 
history and comorbidities), and biological data were collected, and their association with DECT MSU crystal volume was analyzed using bivariate and multivariate analyses. A second bivariate analysis was performed by splitting the dataset depending on an arbitrary threshold of DECT MSU volume $(1 \mathrm{~cm} 3)$.

Results: A total of 125 gout patients were included, of whom 91 underwent both DECT scans of knees and feet. In bivariate analysis, age $(p=0.03)$, symptom duration $(p=0.003)$, subcutaneous tophi $(p=0.004)$, hypertension $(p=0.02)$, diabetes mellitus $(p=0.05)$, and chronic heart failure $(p=0.03)$ were associated with the total DECT volume of MSU crystal deposition. In multivariate analysis, factors associated with DECT MSU volumes $\geq 1 \mathrm{~cm} 3$ were gout duration (OR for each 10-year increase 3.15 [1.60;7.63]), diabetes mellitus (OR 4.75 [1.58;15.63]), and chronic heart failure (OR 7.82 [2.29;31.38]). The model performance was good with an AUC of 0.816 .

Conclusion: Specific comorbidities, particularly chronic heart failure, diabetes mellitus, and hypertension, are more strongly associated with increased MSU crystal deposition in knees and feet than gout duration, regardless of serum urate level.

Disclosure of Interests: : Tristan Pascart Grant/research support from: Research Grant Horizon Pharma, Consultant of: Novartis, BMS, Sanofi, Pfizer,, Speakers bureau: Novartis, BMS, André Ramon: None declared, Sebastien Ottaviani: None declared, Julie Legrand: None declared, Vincent Ducoulombier: None declared, Eric Houvenagel Speakers bureau: Janssen, Novartis, Laurène Norberciak: None declared, Pascal Richette: None declared, Fabio Becce: None declared, Paul Ornetti: None declared, Jean-François Budzik: None declared DOI: 10.1136/annrheumdis-2020-eular.3725

\section{\begin{tabular}{|l|l}
\hline THU0438 INADEQUATE CARE FOR PATIENTS HOSPITALISED \\
\hline
\end{tabular} WITH GOUT: EVIDENCE THAT EULAR GUIDANCE IS NOT UTILISED}

M. Russell ${ }^{1}$, B. Clarke ${ }^{1}$, S. Rampes ${ }^{1}$, K. Dokal ${ }^{1}$, A. Mahto $^{1}$, A. Rutherford ${ }^{1}$, J. Galloway'. ' King's College London, Centre for Rheumatic Diseases, London, United Kingdom

Background: Hospitalisations due to gout have increased over the last decade, in direct contrast to declining admissions for other inflammatory arthritides including RA [1]. Gout is a treatable condition with recently published EULAR guidelines [2, 3]. Admissions could be avoided with effective use of urate-lowering therapies (ULT).

Objectives: We hypothesised that the majority of patients attending hospital with acute gout attacks would not be on ULT. Furthermore, we hypothesised that the majority of patients would not be provided with a plan for ULT commencement and/or uptitration on discharge, leaving them at risk of further hospitalisations.

Methods: We retrospectively analysed electronic health records for all patients presenting acutely with a primary admission diagnosis of gout (ICD-10 code: M10) at two hospitals in London, UK, from January - December 2017. Analyses of in-hospital gout management were performed for these patients, including to ascertain the number and proportion of patients who: i) had a known history of gout; ii) were receiving ULT at time of attendance; iii) were provided with a discharge plan for ULT commencement and/or uptitration.

Results: Over a 12-month period, there were 234 emergency attendances for gout in 225 individuals. $80 \%$ were male, with a mean age of 58 years. 70/234 (30\%) attendances resulted in admission to hospital (mean length of stay: 2 days; range: 0-31 days). 211 patients had routinely captured clinical data available for further analysis. 90/211 (43\%) patients had prior diagnoses of gout, of whom $38 \%$ were on ULT at presentation (32 allopurinol, 2 febuxostat). $38 \%$ of patients were discharged with a plan for ULT commencement and/or uptitration. 20 patients re-presented to hospital with acute gout within 12 months (17/20 were not receiving ULT).

Conclusion: Most patients hospitalised with gout were not receiving ULT, even those with a prior history of gout attacks. Few were provided with a ULT plan, leaving them at risk of re-admission to hospital. Hospital admissions are unpleasant for patients and incur a high economic burden for health services; if they are to be prevented, there must be a concerted effort to implement and follow gout management guidelines to ensure patients receive ULT at appropriate doses. References:

[1] Russell M, et al. Return of the King: Rising Incidence of Acute Hospital Admissions due to Gout. J Rheum 2019 Sep 15.

[2] Richette P, et al. 2016 updated EULAR evidence-based recommendations for the management of gout. Ann Rheum Dis 2017;76:29-42.

[3] Richette P, et al. 2018 updated European League Against Rheumatism evidence-based recommendations for the diagnosis of gout. Ann Rheum Dis 2020;79:31-38.

Disclosure of Interests: : None declared

DOI: 10.1136/annrheumdis-2020-eular.3951

\section{THU0439 \\ EFFICACY AND SAFETY OF ANAKINRA IN THE TREATMENT OF RECURRENT GOUT FLARES: RESULTS FROM THE EXTENSION PHASE OF THE ANAGO STUDY}

K. Saag ${ }^{1}$, P. Khanna ${ }^{2}$, R. Keenan ${ }^{3}$, S. Ohlman ${ }^{4}$, E. Sparve ${ }^{4}$, D. Lindqvist ${ }^{4}$, A. C. Åkerblad ${ }^{4}$, M. Wikén ${ }^{4}$, A. So ${ }^{5}$, M. H. Pillinger ${ }^{6}$, R. Terkeltaub ${ }^{7} .{ }^{1}$ Uni Alabama, Birmingham, United States of America; ${ }^{2}$ Uni Michigan, Ann Arbor, United States of America; ${ }^{3}$ Duke Uni School Med, Durham, United States of America; ${ }^{4}$ Sobi, Stockholm, Sweden; ${ }^{5}$ Uni Lausanne, Lausanne, Switzerland; ${ }^{6}$ New York Uni School Med, New York, United States of America; ${ }^{7}$ Uni California San Diego, La Jolla, United States of America

Background: The anaGO (anakinra in gout) study was a multi-center, randomized, double-blind, double-dummy, phase 2 study investigating the efficacy and safety of anakinra for recurrent gout flares. Results from subsequent flares (extension phase) are presented in relation to the previously reported results from the $1^{\text {st }}$ flare (flare at study enrollment).

Objectives: The objective of the extension phase was to evaluate the efficacy, safety and immunogenicity of two anakinra regimens (100 or $200 \mathrm{mg}$ daily s.c. injections for 5 days) compared to triamcinolone (single i.m. injection $40 \mathrm{mg}$ ) for subsequent flares after initial study enrollment flare. The primary endpoint of the study was change in patient-assessed flare pain intensity from baseline to 24-72 hours (average of 24, 48 and 72 hours) in the most affected joint measured on a visual analogue scale (0-100 VAS). Secondary endpoints included: patient's and physician's assessments of global response, anti-drug antibodies (ADA) and safety. Methods: The study included patients with acute gout (ACR/EULAR 2015 gout classification criteria) unsuitable for anti-inflammatory therapy with NSAIDs and colchicine due to contraindication, intolerance or inefficacy. Patients were eligible for treatment of subsequent flares for up to 2 years. Each patient received the same treatment for all flares, starying with the flare at enrollment.

Results: 161 patients were treated for 1 flare, 61 patients for 2 flares, 31 patients for 3 , and 20 patients for 4 or more flares with 1 patient treated for 9 flares. In total, 300 flares were treated in the full study; anakinra $100 \mathrm{mg}$ and $200 \mathrm{mg}$, 107 and 106 flares, respectively; and triamcinolone, 87 flares. Both anakinra doses and triamcinolone provided a clinical meaningful reduction in patient-assessed pain intensity in both the $1^{\text {st }}$ and subsequent flares. Mean changes in pain intensity from baseline to 24-72 hours for total anakinra and triamcinolone were: $1^{\text {st }}$ flare -41.2 and $-39.4 ; 2^{\text {nd }}$ flare -33.9 and $-31.1 ; 3^{\text {rd }}$ flare -31.8 and -51.2 respectively. Mean differences in pain reduction between anakinra and triamcinolone treatment groups were (negative value favors anakinra): $1^{\text {st }}$ flare $-1.8,2^{\text {nd }}$ flare $-2.83^{\text {rd }}$ flare 19.4 . The majority of secondary endpoints favored anakinra, including patient's and physician's global assessement of response and physician's assessement of the joint. No unexpected safety findings during subsequent flares were identified. 21 patients (19.6\%) developed ADA to anakinra in low titers at some time point; 7 (6.5\%) had pre-existing ADA at baseline and 12 (11.2\%) developed treatment induced ADA. 2 patients had pre-existing ADA to triamcinolone at baseline. 4 patients on anakinra (3.7\%) developed neutralizing antibodies (NAbs). Pre-dose 72 hour anakinra serum concentrations were in similar range for ADA+ and ADA- patients. Presence of ADA was not associated with adverse events or had an impact on pain reduction.

Conclusion: The efficacy and safety of anakinra and triamcinolone in subsequent flares were similar to the findings from $1^{\text {st }}$ flare in patients with acute gout Patient-assessed pain in the $1^{\text {st }}$ and $2^{\text {nd }}$ flare was reduced to similar degrees in all treatment groups, but to a larger extent in the $3^{\text {rd }}$ flare in the small triamcinolone group. Secondary endpoints were in favor of anakinra across flares 1 to 3. The overall incidence of ADA and NAb was low also after repeated anakinra dosing and did not appear to impact exposure, efficacy or safety. In conclusion, anakinra was shown to be an option in the treatment of recurrent gout flares in patients for whom conventional therapy is unsuitable.

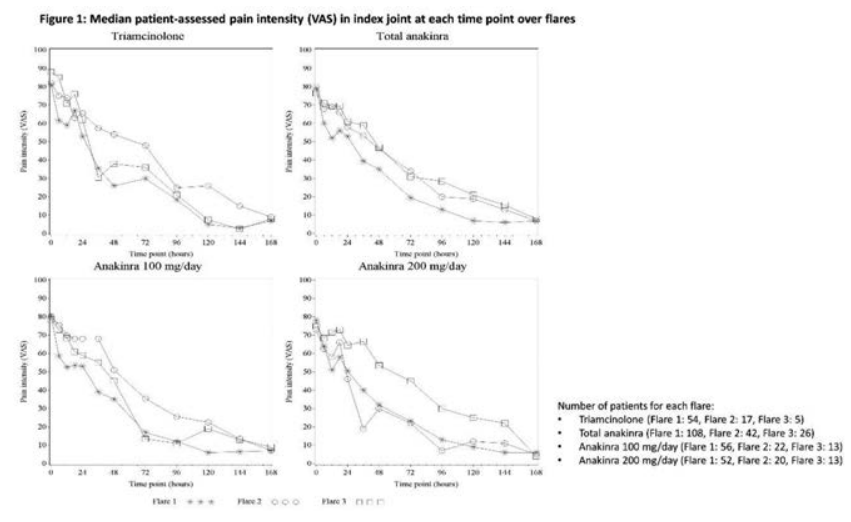

